SLAC-PUB-11785

March 2006

\title{
Using parabolic equation for calculation of beam impedance
}

\author{
Gennady Stupakov \\ Stanford Linear Accelerator Center, Stanford University, Stanford, CA 94309
}

\begin{abstract}
In this paper we develop a new method of parabolic equation (PE) for calculation of both high-frequency and small-angle taper (or collimator) impedances. The applicability of $\mathrm{PE}$ in the high-frequency limit is based on the observation that in this case the contribution to impedance comes from the electromagnetic waves that catch up with the beam far from the obstacle and propagate at small-angles to the axis of the pipe. One of the most important advantages of $\mathrm{PE}$ is that it eliminates the spatial scale of the small wavelength from the problem. As a result, the numerical solution of PE requires coarser spatial meshes.

In the paper we focus on the longitudinal impedance for an axisymmetric geometry and assume a perfect conductivity of the walls. We show how the known analytical results which include a small-angle collimator, step-in and step-out transitions, and a pillbox cavity, can be derived within the framework of the parabolic equation.
\end{abstract}

Submitted for publication to New Journal of Physics 


\section{INTRODUCTION}

Calculation of impedance of various elements of the vacuum system and associated with it beam dynamics effects, such as beam instability or wakefield induced emittance growth, are important elements of the design of a modern accelerator. Sophisticated computer codes are routinely used for such calculations and in many cases can successfully treat complicated geometries of practical problems. There are, however, situations, when simulations require extreme computing resources and long run times.

One such example is the case of very short bunches, envisioned for future linear colliders and light sources. The nominal bunch length in the International Linear Collider (ILC) [1] in the current design is 300 microns, and the rms bunch length in the Linac Coherent Light Source at SLAC [2] will be 20 microns. Since numerical calculation of short-range wake requires spatial mesh size equal to a fraction of the bunch length, submillimeter bunches represent a challenging task for the computations.

Another example where a direct numerical calculation is a difficult problem is related to long, small-angle tapers which are often used to minimize the abruptness of the vacuum chamber transitions. Small-angle collimators will also be used in the post-linac collimation section of the ILC. Numerical solution of Maxwell's equations requires a large mesh covering the full length of the transition or a collimator. The problem becomes especially difficult for short bunches.

Note that the complexity of both problems mentioned above is associated with a presence of a small parameter. For short bunches such a small parameter is the ratio of the bunch length $\sigma_{z}$ to the typical size of the structure in the vacuum chamber $a$ that generates the impedance. If we denote by $\lambda$ the inverse wavenumber $c / \omega$ with $\omega$ being the characteristic frequency of interest for the calculation of the impedance, then $\lambda \sim \sigma_{z}$, and the small parameter of the problem is $\lambda / a$. For a small-angle taper such small parameter is the ratio $a / l$ of the transverse size $a$ of the vacuum chamber to the characteristic length $l$ of the taper.

A small parameter in some cases allows an analytical solution to the problem. For a small-angle taper, Yokoya [3] derived a simple expression for the impedance which is valid in the limit $l \gg a^{2} / \lambda[4]$. Several analytical results are available in the literature for the impedance in the high-frequency limit. They include the impedance of a step transition [5] and the diffraction model for the impedance of a cylindrical cavity [6-9] when the length 
of the cavity satisfies the relation $l_{\text {cav }} \ll b^{2} / \lambda$ with $b$ the radius of the entrance pipe to the cavity. An analytical expression was derived for the impedance of a periodic system of irises [9-12] which represents a long disk-loaded accelerating cavity. Attempts were made to generalize the diffraction theory for other geometries [5] based on an integral equation approach, however, the method of reference [5] works only for some special geometries and, as one can show, does not give the correct numerical factors in the expressions for the impedance.

In this paper we develop a new method of parabolic equation (PE) for calculation of both high-frequency and small-angle taper (or collimator) impedances. Note that these two regimes of applicability of the parabolic equation are complementary - if $a \ll l$ (small-angle approximation) then $\lambda$ can be of order of $a$, and in the limit $\lambda \ll a$ (high-frequency limit) the transverse size $a$ of an obstacle that generates the impedance can be of order of its length $l$.

The applicability of PE in the high-frequency limit is based on the observation that in this case the contribution to impedance comes from the electromagnetic waves that catch up with the beam far from the obstacle and propagate at small-angles to the axis of the pipe. This allows us to simplify the Maxwell equation using the paraxial approximation. The resulting $\mathrm{PE}$ in many cases is much easier to solve than the original equations. Note that PE neglects the backward propagating waves reflected from the obstacle and incorrectly treats the waves propagating at large angles to the axis. Nevertheless, the impedance calculations are correct because those waves do not catch up with the beam and hence do not contribute to the impedance.

The parabolic equation in diffraction theory was proposed many years ago [13] and has been widely used since that time. It is also a standard approximation in the FEL theory [14]. More recently, the paraxial approximation was applied to the beam radiation problems in a toroidal waveguide [15] and in free space [16].

One of the most important advantages of PE is that it eliminates the small wavelength $\lambda$ from the problem. As a result, the numerical solution of PE requires coarser spatial meshes.

In this paper we only focus on the longitudinal impedance for an axisymmetric geometry and assume a perfect conductivity of the walls. Generalization of the method for the transverse impedance calculations will be considered in a separate publication.

The paper is organized as follows. In Section 2 we derive the parabolic equation for an 
axisymmetric electromagnetic field in a round pipe with slowly varying cross section. In Section 3 we apply this equation to the problem of the longitudinal impedance of abrupt transitions in the pipe. In Section 4 we study a small-angle collimator and obtain Yokoya's result for the longitudinal impedance in the limit $l \gg a^{2} / \lambda$. In Section 5 a numerical solution for a small-angle collimator is presented when $l \sim a^{2} / \lambda$, and in Section 6 the impedance of a pillbox cavity is derived. In Section 7 we discuss the main results of the paper.

We use the Gaussian system of units throughout this paper.

\section{DERIVATION OF THE PARABOLIC EQUATION FOR CALCULATION OF THE LONGITUDINAL IMPEDANCE}

We consider an axisymmetric geometry of a pipe with a varying radius and a straight axis which is chosen as the $z$ axis of the cylindrical coordinate system. The pipe wall is given by the equation $r=a(z)$. The beam is represented by a filament current $I$ on the axis of the pipe propagating with the speed of light:

$$
I(z, t)=I_{0} e^{i k(z-c t)},
$$

where $I_{0}$ is the amplitude of the current, and $k=\omega / c$, with $c$ being the speed of light. Due to the factor $e^{-i k c t}$ in this equation all the fields in the system will also have the time dependence $e^{-i \omega t}$ which we will omit in the subsequent equations. Solving Maxwell's equation with the source given by Eq. (1) and the perfectly conducting boundary conditions on the wall, one can find the longitudinal electric field $E_{z}(r, z)$. This field taken on the axis of the pipe and integrated with the factor $e^{-i k z}$ gives the longitudinal impedance at the frequency $\omega$ :

$$
Z(\omega)=-\left.\frac{1}{I_{0}} \int_{-\infty}^{\infty} d z e^{-i k z} E_{z}\right|_{r=0}
$$

We will use the following representation for the components of the electric field

$$
E_{r}(r, z)=\hat{E}_{r}(r, z) e^{i k z}, \quad E_{z}(r, z)=\hat{E}_{z}(r, z) e^{i k z}
$$

where the dependence of the functions $\hat{E}_{z}$ and $\hat{E}_{r}$ is considered as a "slow" one, which means that $\left|\partial \ln \hat{E}_{z} / \partial z\right| \ll k,\left|\partial \ln \hat{E}_{r} / \partial z\right| \ll k$. Introducing the characteristic length scale $l$ through $l^{-1} \sim\left|\partial \ln \hat{E}_{z} / \partial z\right|$ we require $l$ to be much larger than the reduced wavelength

入. For a small-angle taper the length $l$ will be associated with the transition length. For 
the high-frequency calculations, as we will see below, $l \sim k a^{2}$, and the condition $l \gg \lambda$ is satisfied if $k a \gg 1$.

It is convenient to subtract from the field $\hat{E}_{r}$ the vacuum field of the beam

$$
\hat{E}_{r}=\frac{2 I_{0}}{r c}+\mathcal{E} .
$$

The field $\mathcal{E}$ can be considered as generated by the image charges and currents in the walls. It satisfies the following equation

$$
\frac{1}{r} \frac{\partial}{\partial r} r \frac{\partial \mathcal{E}}{\partial r}-\frac{\mathcal{E}}{r^{2}}+\frac{\partial^{2} \mathcal{E}}{\partial z^{2}}-\frac{\omega^{2}}{c^{2}} \mathcal{E}=0
$$

(the field $\hat{E}_{r}$ satisfies the same equation with the source on the right hand side due to the beam). Substituting Eq. (3) into this equation and neglecting the term $\partial^{2} \mathcal{E} / \partial z^{2}$ in comparison with $k \partial \mathcal{E} / \partial \zeta$ we obtain the parabolic equation for $\mathcal{E}$,

$$
\frac{1}{r} \frac{\partial}{\partial r} r \frac{\partial \mathcal{E}}{\partial r}-\frac{\mathcal{E}}{r^{2}}=-2 i k \frac{\partial \mathcal{E}}{\partial z}
$$

As was mentioned in the introduction, a remarkable feature of this equation is that the wavenumber $k$ can be eliminated from it by changing the longitudinal coordinate $z \rightarrow z / k$. With this substitution a small wavelength $2 \pi / k$ associated with the frequency $\omega$ is eliminated from the problem.

Using equation $\nabla \cdot \vec{E}=0$, in which we approximately set $\partial E_{z} / \partial z \approx i k E_{z}$, we can express the longitudinal component of the electric field through the radial one,

$$
\hat{E}_{z}=\frac{i}{k} \frac{1}{r} \frac{\partial}{\partial r} r \hat{E}_{r}=\frac{i}{k} \frac{1}{r} \frac{\partial}{\partial r} r \mathcal{E} .
$$

The boundary condition for Eq. (6) consists in the requirement that the tangential field on the surface of the pipe vanishes, $\hat{E}_{t}=0$ at $r=a(z)$. The tangential component $\hat{E}_{t}$ in each particular case can be expressed in terms of $\hat{E}_{r}$ and $\hat{E}_{z}$. Since $\hat{E}_{z}$ is related to $\mathcal{E}$ through Eq. (7) the boundary condition in general case involves both $\mathcal{E}$ and $\partial \mathcal{E} / \partial r$ on the wall.

\section{STEP-IN AND STEP-OUT TRANSITIONS}

In this Section we consider an abrupt transition from a pipe of radius $b$ to radius $a$. Following terminology of Ref. [5] we will call the case $b>a$ the step-in transition, and the opposite case the step-out, see Fig. 1. We will apply PE to the calculation of impedances for these transitions, starting with the case of the step-out. 

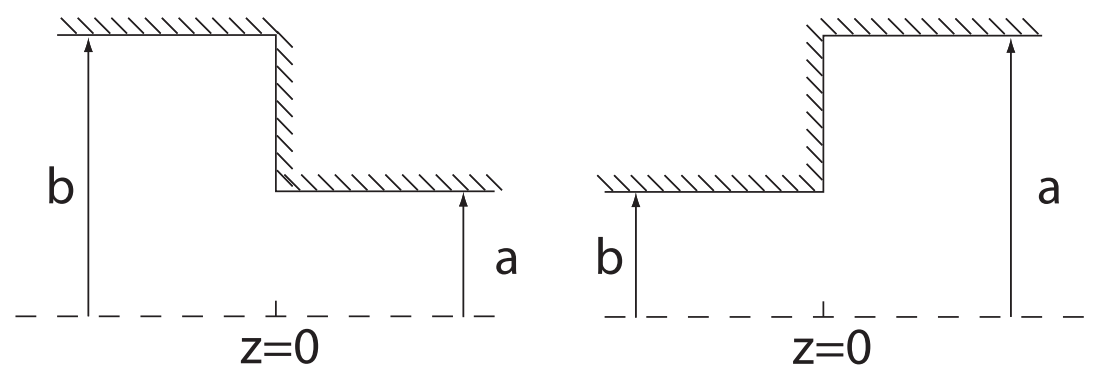

FIG. 1: Step-in (left) and step-out (right) transitions. The beam propagates from left to right. The origin of the coordinate system is chosen at the location of the step.

To solve Eq. (6) in the region $z>0$ and $0<r<a$ it is convenient to introduce the function $\psi=r \mathcal{E}$ and dimensionless variables $\rho=r / a$ and $\xi=z / 2 k a^{2}$. The function $\psi(\rho, \xi)$ satisfies the following equation:

$$
-i \frac{\partial \psi}{\partial \xi}=\rho \frac{\partial}{\partial \rho} \frac{1}{\rho} \frac{\partial \psi}{\partial \rho}
$$

The boundary condition at $\rho=1$ for this equation follows from the requirement $\hat{E}_{z}=0$ at the wall of the pipe, which, using Eq. (7), gives

$$
\left.\frac{\partial \psi}{\partial \rho}\right|_{\rho=1}=0 .
$$

We will also need an initial condition for the function $\psi$ at $z=0, \psi(\rho, 0)=\psi_{0}(\rho)$. The function $\psi_{0}(\rho)$ is determined from the following consideration. In the region $z<0$ and $r<b$, which is a straight pipe with perfectly conducting walls, the field is equal to the beam field in free space, and hence $\mathcal{E}=0$. Because of continuity of $\mathcal{E}, \mathcal{E}=0$ at $z=0$, and hence $\psi_{0}(\rho)=0$ for $\rho<b / a$. The requirement that the radial electric field at the vertical surface of the metal at $z=0$ vanishes means that $\mathcal{E}=-2 I_{0} / r c$ at $z=0$ and $b<r<a$. These two conditions define the function $\psi_{0}$ at $\xi=0$ :

$$
\psi_{0}=\left\{\begin{array}{c}
-2 I_{0} / c, b / a<\rho<1, \\
0, b / a>\rho .
\end{array}\right.
$$

The solution of Eq. (8) can be written as

$$
\psi(\rho, \xi)=\sum_{n=1}^{\infty} c_{n} e^{-i \nu_{n}^{2} \xi} \rho J_{1}\left(\nu_{n} \rho\right),
$$


and the boundary condition (9) is satisfied if $\nu_{n}$ are the roots of the Bessel function of the zeroth order, $J_{0}\left(\nu_{n}\right)=0$. The coefficients $c_{n}$ are found from the initial condition using the orthogonality relations for the Bessel functions:

$$
c_{n}=\frac{2}{J_{1}\left(\nu_{n}\right)^{2}} \int_{0}^{1} d \rho \psi_{0}(\rho) J_{1}\left(\nu_{n} \rho\right) .
$$

For the function $\psi_{0}$ given by Eq. (10) the integration can be carried out analytically with the result

$$
c_{n}=-\frac{4 I_{0}}{c} \frac{J_{0}\left(\nu_{n} b / a\right)}{\nu_{n} J_{1}\left(\nu_{n}\right)^{2}} .
$$

We can now calculate the longitudinal impedance using Eq. (2)

$$
Z=-\frac{1}{I_{0}} \int_{0}^{\infty} e^{-i k z} E_{z}(r=0, z) d z=-\left.\frac{2 i}{I_{0}} \int_{0}^{\infty} d \xi\left(\frac{1}{\rho} \frac{\partial \psi}{\partial \rho}\right)\right|_{\rho \rightarrow 0}
$$

Using Eqs. (11) and (13) one finds,

$$
Z=\frac{8}{c} \sum_{n=1}^{\infty} \frac{J_{0}\left(\nu_{n} b / a\right)}{\nu_{n}^{2} J_{1}\left(\nu_{n}\right)^{2}},
$$

and using the mathematical identity

$$
\sum_{n=1}^{\infty} \frac{J_{0}\left(\nu_{n} / x\right)}{\nu_{n}^{2} J_{1}\left(\nu_{n}\right)^{2}}=\frac{1}{2} \ln x,
$$

valid for $x>0$ we arrive at the following expression for $Z$

$$
Z=\frac{4}{c} \ln \frac{a}{b}=\frac{Z_{0}}{\pi} \ln \frac{a}{b}
$$

where $Z_{0}=4 \pi / c=377 \mathrm{Ohm}$. This is a well known result for the longitudinal impedance for the step-out in the limit of high frequencies [5]. This impedance is real and corresponds to the energy loss of the beam when it builds up the electromagnetic field in the region of the expanded pipe.

In the case of a step-in transitions, the initial condition $\psi_{0}=0$ at $r<a$ means that the solution of the parabolic equation in the region $z>0$ is identically equal to zero, $\psi=0$, and hence $Z=0$. The step-in transition does not generate impedance in the limit of high frequencies which is also a well known result [5]. Note that solving PE for the step-in geometry we ignore the nonzero tangential electric field at the vertical part of the wall at $z=0$. This boundary condition results in the field which is reflected by the step in the backward direction. Since the backward propagating waves are not accounted for by the 
parabolic equation, they are being ignored in the calculation based on PE. It is important however that in the limit of high frequencies those waves never catch up and interact with the beam and hence do not contribute to the impedance.

A sequence of step-in and step-out transitions following each other makes a cylindrical collimator-like protrusion into the pipe. To calculate the impedance of such a protrusion, we have to combine the results derived above for the step-in and step-out. Since the impedance of the step-in is zero the impedance of such a protrusion is equal to that of the step-out. Remarkably, it does not depend on the length of the protrusion.

\section{SMALL-ANGLE TRANSITIONS}

In this section we will consider a small-angle transitions shown in Fig. 2. The pipe radius

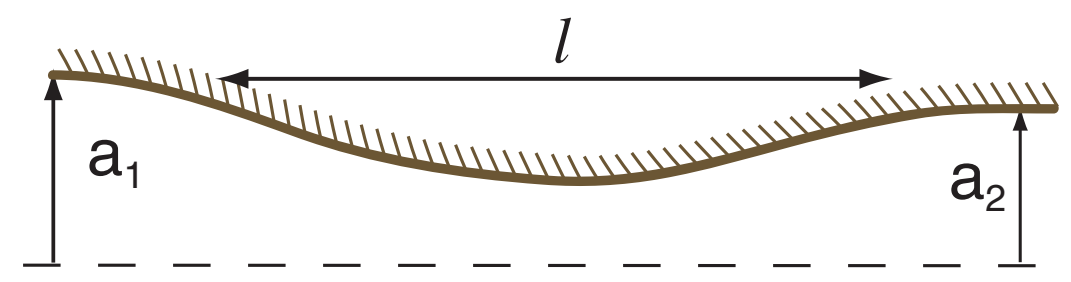

FIG. 2: A small-angle transition from a round pipe of radius $a_{1}$ to $a_{2}$. The dashed line indicates the axis of the pipe.

$a(z)$ varies from the value $a_{1}$ on the far left end to the value $a_{2}$ on the far right. The smallangle approximation means that $|d a / d z| \ll 1$. The tangential component of the electric field on the wall should be equal to zero, which approximately can be written as

$$
\left.\hat{E}_{t} \approx\left(\hat{E}_{z}+a^{\prime} \hat{E}_{r}\right)\right|_{r=a(z)}=\left.\frac{i}{k a} \frac{\partial}{\partial r} r \mathcal{E}\right|_{r=a(z)}+a^{\prime} \mathcal{E}+\frac{2 I_{0} a^{\prime}}{a c}=0
$$

where $a^{\prime}=d a / d z$. We introduce the function $\psi=r \mathcal{E}$ and the new variables $\rho=r / a(z)$ and $\xi=z / 2 k a_{0}^{2}$, where $a_{0}$ is some characteristic value for the pipe radius. We also introduce the ratio $\alpha=a(z) / a_{0}$ and consider it as a function of $\xi$ with $\alpha_{\xi}$ denoting the derivative $d \alpha / d \xi$. From Eq. (6) it follows that the function $\psi(\rho, \xi)$ satisfies the following equation

$$
\rho \frac{\partial}{\partial \rho} \frac{1}{\rho} \frac{\partial \psi}{\partial \rho}=-i \alpha\left(\alpha \frac{\partial \psi}{\partial \xi}-\rho \alpha_{\xi} \frac{\partial \psi}{\partial \rho}\right)
$$

The boundary condition (18) translates into the following one for the function $\psi$

$$
\left.2 \frac{\partial \psi}{\partial \rho}\right|_{\rho=1}-i \alpha_{\xi} \alpha \psi=i \alpha_{\xi} \alpha \frac{2 I_{0}}{c} .
$$


We can solve this equation analytically in the limit of a very long transition, when the characteristic length of the transition $l$ is such that $l \gg k a^{2}$, or $\left|\alpha_{\xi}\right| \ll 1$. In this limit the right hand side of Eq. (19) can be considered as a perturbation, and in the zeroth approximation the equation is simplified to

$$
\rho \frac{\partial}{\partial \rho} \frac{1}{\rho} \frac{\partial \psi_{0}}{\partial \rho}=0
$$

and the boundary condition Eq. (20) becomes

$$
\left.\frac{\partial \psi_{0}}{\partial \rho}\right|_{\rho=1}=i \alpha_{\xi} \alpha \frac{I_{0}}{c}
$$

The solution of these two equations is

$$
\psi_{0}=i \alpha_{\xi} \alpha \frac{I_{0}}{2 c} \rho^{2}
$$

Eq. (2) can be written as

$$
Z=-\left.\frac{2 i}{I_{0}} \int_{-\infty}^{\infty} \frac{d \xi}{\alpha^{2}}\left(\frac{1}{\rho} \frac{\partial \psi}{\partial \rho}\right)\right|_{\rho \rightarrow 0},
$$

which upon substitution of Eq. (23) gives

$$
Z^{(0)}=\frac{2}{c} \ln \frac{a_{2}}{a_{1}}=\frac{Z_{0}}{2 \pi} \ln \frac{a_{2}}{a_{1}} .
$$

We see that in this approximation the impedance is real and depending on the ratio $a_{2} / a_{1}$ can be either positive of negative. The negative value of the real part of the impedance means that the beam gains energy from the electromagnetic field.

For $a_{1}=a_{2}$ the lowest order calculation Eq. (25) vanishes and we need to make one more iteration in the small parameter $\alpha_{\xi}$. We seek solution $\psi=\psi_{0}+\psi_{1}$, with $\left|\psi_{1}\right| \ll\left|\psi_{0}\right|$, where $\psi_{0}$ denotes the function $\psi$ found above in the first order (proportional to $\left|\alpha_{\xi}\right|$ ), and $\psi_{1}$ is of the second order. The equation for $\psi_{1}$ reads

$$
\rho \frac{\partial}{\partial \rho} \frac{1}{\rho} \frac{\partial \psi_{1}}{\partial \rho}=-i \alpha\left(\alpha \frac{\partial \psi_{0}}{\partial \xi}-\rho \alpha_{\xi} \frac{\partial \psi_{0}}{\partial \rho}\right)=\rho^{2} \frac{I_{0}}{2 c} \alpha^{4} \frac{d}{d \xi}\left(\frac{\alpha_{\xi}}{\alpha}\right)
$$

and the boundary condition for $\psi_{1}$ is obtained as a second order approximation in Eq. (20)

$$
\left.\frac{\partial \psi_{1}}{\partial \rho}\right|_{\rho=1}=-\left(\alpha \alpha_{\xi}\right)^{2} \frac{I_{0}}{4 c} .
$$

The solution of the above two equations is

$$
\psi_{1}=\frac{1}{16} \rho^{4} \alpha^{4} \frac{I_{0}}{c} \frac{d}{d \xi}\left(\frac{\alpha_{\xi}}{\alpha}\right)+\frac{1}{2} B \rho^{2},
$$


with

$$
B=-\frac{I_{0}}{4 c}\left(\alpha^{4} \frac{d}{d \xi}\left(\frac{\alpha_{\xi}}{\alpha}\right)+\left(\alpha \alpha_{\xi}\right)^{2}\right)
$$

Putting $\psi_{1}$ into Eq. (24) for the impedance we find

$$
Z^{(1)}=-\frac{2 i}{I_{0}} \int_{-\infty}^{\infty} \frac{d \xi}{\alpha^{2}} B=-\frac{i}{2 c} \int_{-\infty}^{\infty} d \xi \alpha_{\xi}^{2}=-\frac{i k}{c} \int d z a^{\prime 2}
$$

with the total impedance

$$
Z=Z^{(0)}+Z^{(1)}
$$

This result was first obtained in Ref. [3]. Note that the second order term $Z^{(1)}$ describes a purely inductive part of the total impedance.

\section{NUMERICAL SOLUTION FOR A SMALL-ANGLE COLLIMATOR}

The analytical result derived in the previous section is only valid in the limit $l \gg k a^{2}$. In a more general case when the parameter $l / k a^{2}$ is of order of one, one has to invoke numerical methods to solve the parabolic equation. In this Section we will show an example of such a solution for a particular geometry of a collimator given by the equation

$$
a(z)=a_{0}\left(1-A \sin ^{4} \frac{z}{L_{\mathrm{coll}}}\right)
$$

where $L_{\text {coll }}$ is the parameter that determines the length of the collimator. The value of $A$ for this calculation was chosen $A=0.5$.

For numerical solution it is convenient to use the following variables

$$
\zeta=\frac{z}{k a_{0}^{2}}, \quad \phi=i \psi \frac{c}{I_{0}}, \quad v=\frac{1}{2} \frac{r^{2}}{a(z)^{2}}, \quad \tau=\frac{a(z)^{2}}{a_{0}^{2}},
$$

and consider $\tau$ as a function of $\zeta$, and $\phi$ as a function of $\zeta$ and $v, \phi(\zeta, v)$, defined in the region $0<v<1 / 2$. Then Eq. (19) takes the form

$$
\frac{\partial \phi}{\partial \zeta}=\frac{i}{\tau(\zeta)} v \frac{\partial^{2} \phi}{\partial v^{2}}+\frac{\tau_{\zeta}(\zeta)}{\tau(\zeta)} v \frac{\partial \phi}{\partial v},
$$

and the boundary condition (20) becomes

$$
\left.\frac{\partial \phi}{\partial v}\right|_{v=1 / 2}-\left.\frac{1}{2} i \tau_{\zeta}(\zeta) \phi\right|_{v=1 / 2}=-\tau_{\zeta}(\zeta)
$$


where $\tau_{\zeta}=d \tau / d \zeta$. Note also that because the radial electric field on the axis is equal to zero, we have

$$
v(\zeta, 0)=0
$$

The equation for the collimator wall given by Eq. (32) in terms of the new variables becomes

$$
\tau=\left(1-A \sin ^{4} \frac{\pi \zeta}{\ell}\right)^{2}
$$

where $\ell=\pi L_{\text {coll }} / k a_{0}^{2}$.

Eq. (34) with the boundary conditions (35) and (36) can be rather easily solved numerically with existent standard PDE solvers[19]. The color plot of this solution is shown in
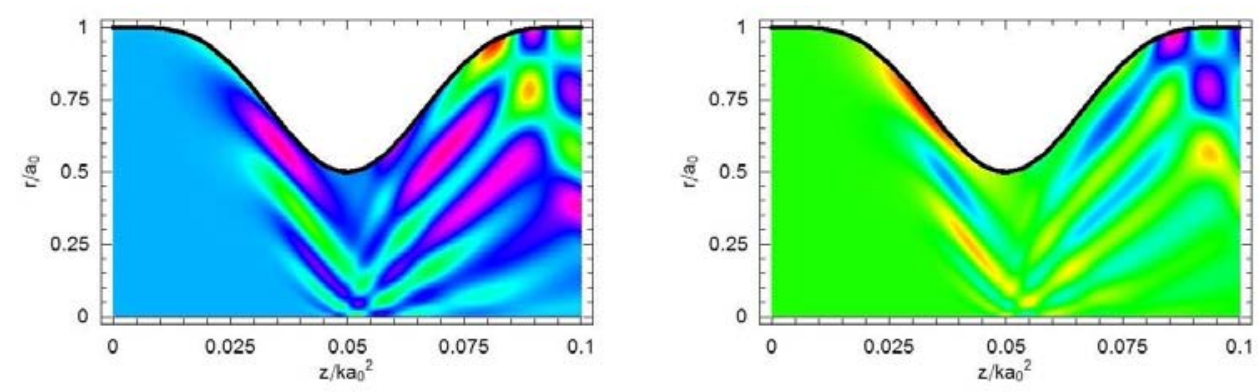

FIG. 3: Real (left) and imaginary (right) parts of the solution of Eq. (34) for $\ell=0.1$. The color coding goes from magenta to blue, green and red when the value of the function varies from the minimum to the maximum. The black curve shows the wall of the collimator.

Fig. 3 for a short collimator with $\ell=0.1$. The interference pattern observed on this plot is due to the waves reflected from the walls of the converging part of the collimator and focusing at some point on the axis of the system.

To calculate the impedance of the collimator we use Eq. (24) which for the new variables can be written as

$$
Z=-\left.\frac{1}{c} \int_{-\infty}^{\infty} \frac{d \zeta}{\tau(\zeta)} \frac{\partial \phi}{\partial v}\right|_{v=0}
$$

Note that the region $\zeta<0$, preceding the entrance to the collimator, does not contribute to this integral. The contribution from the interval $0<\zeta<\ell$ was calculated with the numerically found solution $\phi$. The value of this solution at $\zeta=\ell$ was also used as an initial condition for the straight pipe $\zeta>\ell$. Using the method described in Section 1 the contribution from this area was computed analytically. 


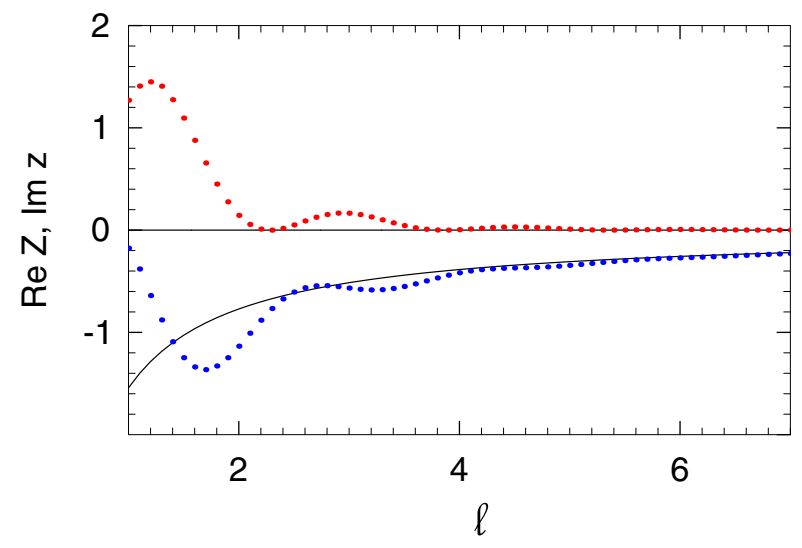

FIG. 4: Real (red) and imaginary (blue) part of the collimator impedance normalized by $Z_{0} / 4 \pi$. The black line is the theoretical value for a long collimator given by Eq. (30).

The resulting total impedance for the collimator is shown in Figs. 4 and 5 as a function of the normalized collimator length $\ell$. Fig. 4 shows also the plot of the imaginary part of the impedance given by Eq. (30) and valid in the limit $\ell \gg 1$. As we see from this plot, the numerically computed values agree well with this curve for $\ell \gtrsim 3$. Fig. 5 corresponding to small values of $\ell$ demonstrates an oscillatory behavior as $\ell$ approaches zero. One can see

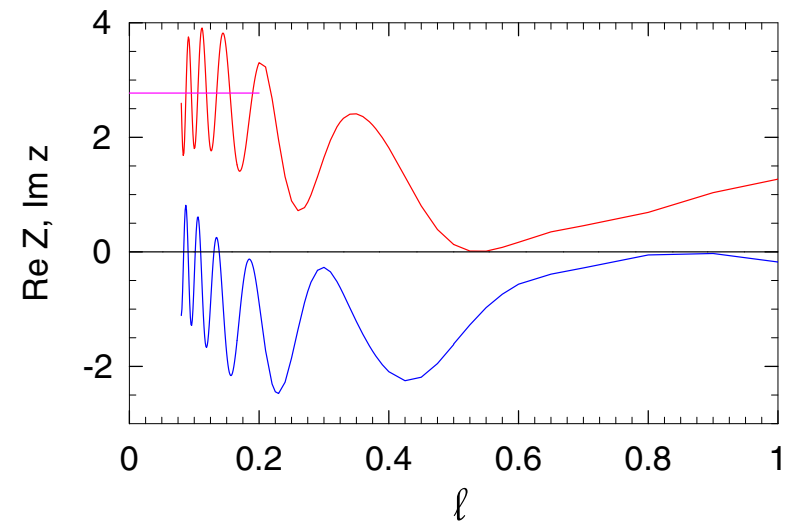

FIG. 5: Real (red) and imaginary (blue) part of the collimator impedance normalized by $Z_{0} / 4 \pi$. The horizontal line (magenta) shows the theoretical value of the real part of the impedance for an iris.

that averaged over these oscillations, the imaginary part tends to zero, and the real part approaches a constant value. We expect this constant value to be equal to the impedance of an iris of radius $(1-A) a_{0}$. Indeed, the impedance of such an iris given by Eq. (17) with $b=a_{0}(1-A)$ and shown on the plot by a horizontal line, demonstrates a good agreement 
with the limiting value of $\operatorname{Re} Z$.

\section{PILLBOX CAVITY}

We will now calculate the impedance of a cylindrical cavity of radius $a$ and length $L$ shown in Fig. 6. The entrance and exit pipes are of radius $b$. This problem is a combination of step-out and step-in geometries, and we can aplly the solution developed in Section 1 to this geometry. In what follows we will use the dimensionless cavity length $\mathcal{L}=L / 2 k a^{2}$.

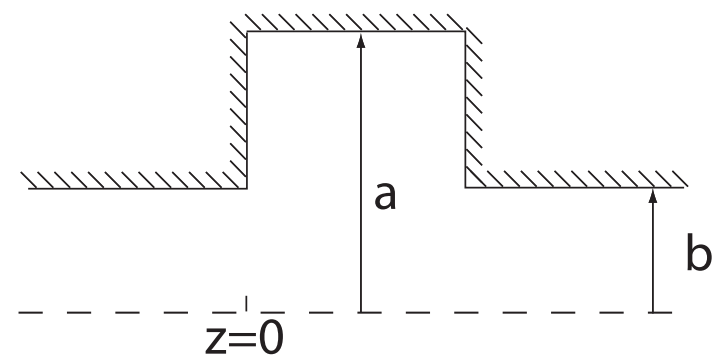

FIG. 6: A pillbox cavity. The length of the cavity is $L$. The origin of the coordinate system is chosen at the entrance to the cavity.

We can calculate the impedance of the cavity as consisting of two parts. The first one, $Z_{1}$, is the contribution to the integral Eq. (2) from the cavity region $0<z<L$, and the second one, $Z_{2}$, comes from the region $L<z$, so that

$$
Z=Z_{1}+Z_{2}
$$

The calculation of the first part follows exactly along the path described in the previous section with the only difference that the integration in Eq. (14) is carried out over the distance $0<z<L$, with the result

$$
Z_{1}=-\frac{2 i}{I_{0}} \sum_{n=1}^{\infty} \nu_{n} c_{n} \int_{0}^{2 k L a^{2}} d \xi e^{i \nu_{n}^{2} \xi}=\frac{8}{c} \sum_{n=1}^{\infty} \frac{J_{0}\left(\nu_{n} b / a\right)}{\nu_{n}^{2} J_{1}\left(\nu_{n}\right)^{2}}\left(1-e^{-i \nu_{n}^{2} \mathcal{L}}\right)
$$

Putting $\xi=\mathcal{L}$ in Eq. (11) gives the function $\psi$ in the cross section at the exit from the cavity,

$$
\psi(\rho, \xi=\mathcal{L})=\sum_{n=1}^{\infty} c_{n} e^{-i \nu_{n}^{2} \mathcal{L}} \rho J_{1}\left(\nu_{n} \rho\right), \quad 0<\rho<b / a
$$


To find the function $\psi$ in the region $\xi>\mathcal{L}$ one needs to solve the parabolic equation (8) with the initial condition given by Eq. (41) and the boundary condition

$$
\left.\frac{\partial \psi}{\partial \rho}\right|_{\rho=b / a}=0,
$$

where the coefficients $c_{n}$ are given by Eq. (13). Solving this equation by the method described in the previous section and calculating the impedance $Z_{2}$, gives the following result

$$
Z_{2}=\frac{16}{c}\left(\frac{b}{a}\right)^{2} \sum_{n=1}^{\infty} \frac{1}{\nu_{n} J_{1}\left(\nu_{n}\right)} \sum_{m=1}^{\infty} \frac{J_{0}\left(\nu_{m} b / a\right)^{2}}{J_{1}\left(\nu_{m}\right)^{2}\left(\nu_{n}^{2}-b^{2} / \nu_{m}^{2} a^{2}\right)} e^{-i \nu_{m}^{2} \mathcal{L}}
$$

Note that this expression is valid for arbitrary relations between $L, a$ and $b$.

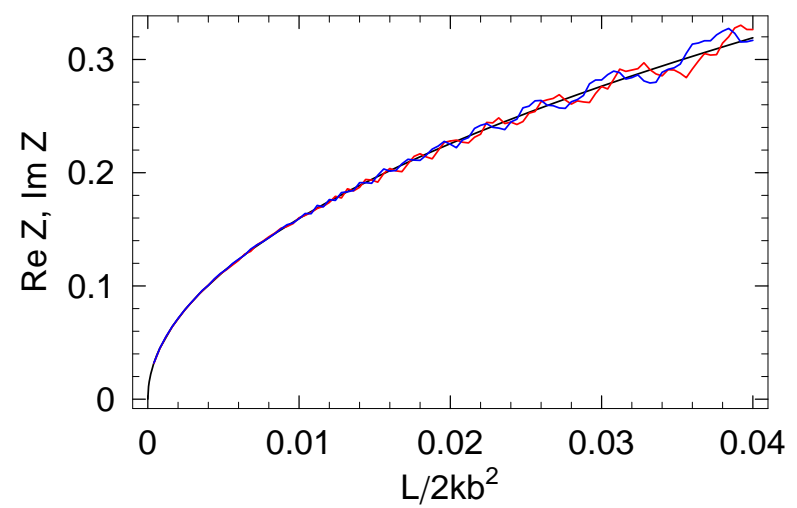

FIG. 7: Real (red) and imaginary (blue) part of the impedance of a short pillbox cavity in comparison with the diffraction model (black). The impedance is normalized by $Z_{0} / 4 \pi$.

In the limit of short cavity, $L \ll k b^{2}$, the impedance of the pillbox cavity is given by the diffraction model [8],

$$
Z_{\text {diff }}=\frac{2(1+i)}{\sqrt{\pi} b} \sqrt{\frac{c L}{\omega}},
$$

and is independent of the value of $a$. To compare our result with this equation we calculated the impedance $Z_{1}+Z_{2}$ for $b / a=0.5$ for small values of the parameter $\mathcal{L}$ truncating the infinite sums in Eqs. (40) and (43) to 200 terms. The result is shown in Fig. 7 and indeed demonstrates a good agreement with the diffraction model in the limit $L \ll k b^{2}$, as well as deviations from this limit as $L$ increases.

The impedance for a long cavity as a function of $L / k a^{2}$ (for the same ratio $a / b=2$ ) is shown in Fig. 8. One might expect that as the length of the cavity $L$ increases, the 


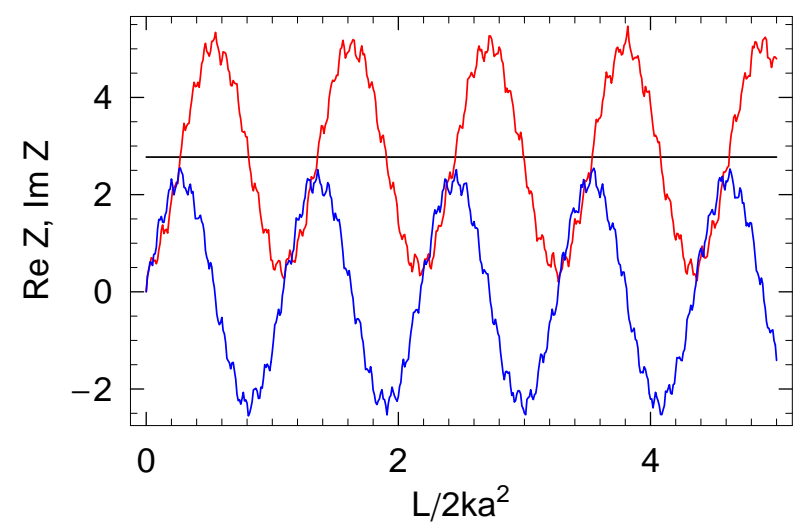

FIG. 8: Real (red) and imaginary (blue) part of the impedance (in units $Z_{0} / 4 \pi$ ) of a long pillbox as a function of the normalized length $L / 2 k a^{2}$. The horizontal line shows the value of the real part of the impedance given by Eq. (17).

impedance should tend to the value given by the sum of the impedances of two stepsstep-out an step-in. Since the impedance of step-in is equal to zero, this sum is given by Eq. (17). However, as is seen from Fig. 8 this occurs only in average - the impedance of the cavity oscillates around a value that agrees with Eq. (17).

We also compared the results for the pillbox cavity obtained through solution of PE with direct numerical simulations using the ABCI computer code [18]. The code was run with the following dimensions of the pillbox cavity: $a=10 \mathrm{~cm}, b=5 \mathrm{~cm}$ and $L=400 \mathrm{~cm}$, and the impedance was calculated in the frequency range up to $40 \mathrm{GHz}$. The results of these calculations for the real and imaginary part of the impedance are shown in Fig. 9. Apart from the fast oscillations in the numerical solution it is seen that the numerically calculated impedance on average agrees with the solution of PE.

\section{CONCLUSIONS}

In this paper we presented several results of impedance calculation using an approach based on the parabolic equation. We showed how the known analytical results can be derived within the framework of PE. These include a small-angle collimator, step-in and step-out transitions, and a pillbox cavity.

For the pillbox cavity, our result (Eqs. (40) and (43)) extends the diffraction theory 

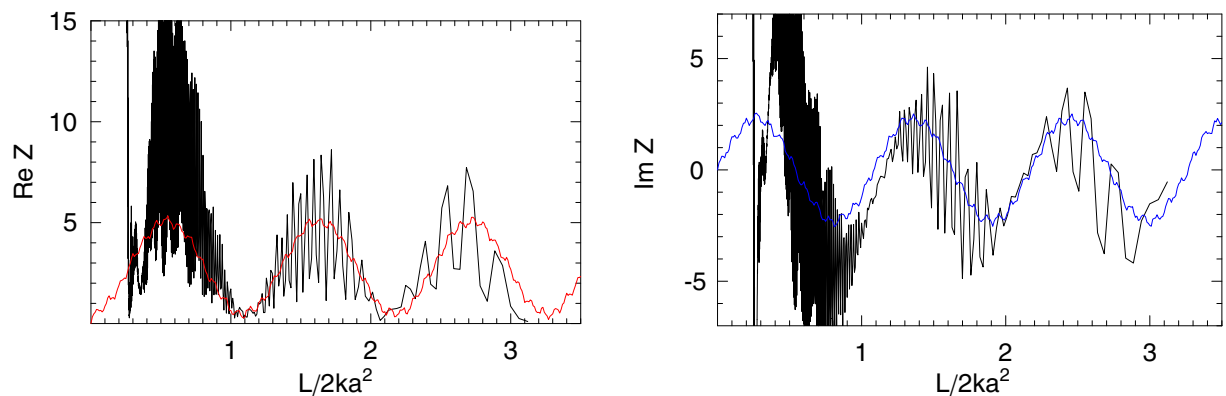

FIG. 9: Comparison of ABCI results for a long pillbox (black line) with the solution of the parabolic equation (red and blue lines for the real and imaginary parts, respectively).

valid in the limit $L \ll k b^{2}$ to the case of arbitrary length of the cavity. It agrees with the diffraction model in the limit of a short cavity, and is confirmed for the case of a long cavity by comparison with direct numerical calculations using the ABCI code.

For the small-angle collimator, we also obtained a numerical solution of PE using a standard partial differential equation solver. This numerical solution agrees with Yokoya's formula in the limit of very large $l$ and tends to the impedance of an iris in the limit $l \ll a^{2} / \lambda$.

PE can also be used for calculation of the impedance of a periodic system of irises in a round pipe (a model of disk loaded accelerated structure). The solution of this problem agrees with the previous result which can be found in the literature [6-9]. This problem, however, is beyond the scope of the present article and will be published in a separate paper.

\section{Acknowledgments}

I would like to thank S. Heifets for useful discussions. This work was supported by Department of Energy contract DE-AC02-76SF00515.

\section{References}

[1] K. Yokoya. GG1 summary talk. In 2005 International Linear Collider Physics and Detector Workshop and Second ILC Accelerator Workshop, Snowmass, CO, 2005. 
[2] Linac Coherent Light Source (LCLS) conceptual design report. Report SLAC-R-593, SLAC, 2002.

[3] K. Yokoya. Impedance of slowly tapered structures. Technical Report SL/90-88 (AP), CERN, 1990.

[4] G.V. Stupakov. Geometrical wake of a smooth taper. Particle Accelerators, 56(2):83-97, 1996.

[5] S. A. Heifets and S. A. Kheifets. Coupling impedance in modern accelerators. Review of Modern Physics, 63:631, 1991.

[6] J. D. Lawson. Radiation from a ring charge passing through a resonator. Part. Accel., 25:107, 1990.

[7] R. B. Palmer. A qualitative study of wake fields for very short bunches. Part. Accel., 25:97, 1990.

[8] Karl Bane and Matthew Sands. Wakefields of very short bunches in an accelerating cavity. Part. Accel., 25:73, 1990.

[9] S. A. Heifets and S. A. Kheifets. High-frequency limit of the longitudinal impedance. Part. Accel., 25:61, 1990.

[10] E. Keil. Diffraction radiation of charged rings moving in a corrugated cylindrical pile. Nucl. Instr. Meth., 100:419-427, 1972.

[11] R. K. Gluckstern. Longitudinal impedance of a periodic structure at high frequency. Phys. Rev., D39:2780, 1989.

[12] G.V. Stupakov. Coupling impedance of a periodic array of diaphragms. In Proceedings of the 1995 Particle Accelerator Conference, volume 5, pages 3303-5, Piscataway, NJ, 1995.

[13] M. Lentovich and V. Fock. Solution of the problem of propagation of electromagnetic waves along the Earth's surface by the method of parabolic equation. Journal of Physics, 10:13, 1946.

[14] E. L. Saldin, E. A. Schneidmiller, and M. V. Yurkov. The Physics of Free Electron Lasers. Springer, 2000.

[15] T. Agoh and K. Yokoya. Calculation of coherent synchrotron radiation using mesh. Phys. Rev. ST Accel. Beams, 7:054403, 2004.

[16] Gianluca Geloni, Evgeni Saldin, Evgeni Schneidmiller, and Mikhail Yurkov. Paraxial Green's functions in synchrotron radiation theory. Report 05-032, DESY, 2005.

[17] S. Wolfram. The Mathematica Book. Wolfram Media/Cambridge University Press, 4th edition, 
1999.

[18] Yong Ho Chin. User's guide for ABCI. Report 2005-06, KEK, 2005.

[19] For results presented in this paper we used the differential equations solver NDSolve from the Mathematica package [17]. 